\title{
Peer-Aware Collaborative Access Control in Social Networks
}

\author{
(Invited Paper) \\ Qian Xiao \\ School of Computing and \\ NUS Graduate School for \\ Integrated Sciences and Engineering \\ National University of Singapore \\ Singapore \\ Email: xiaoqian@nus.edu.sg \\ Kian-Lee Tan \\ School of Computing and \\ NUS Graduate School for \\ Integrated Sciences and Engineering \\ National University of Singapore \\ Singapore \\ Email: tankl@comp.nus.edu.sg
}

\begin{abstract}
Todays online social networks (OSNs) allow a user to share his photos with others and tag the co-owners, i.e., friends who also appear in the co-owned photos. However, it is not uncommon that conflicts may arise among the co-owners because of their different privacy concerns. OSNs, unfortunately, offer only limited access control support where the publisher of the shared content is the sole decision maker to restrict access. There is thus an urgent need to develop mechanisms for multiple owners of the shared content to collaboratively determine the access rights of other users, as well as to resolve the conflicts among coowners with different requirements. Rather than competing with each other and just wanting ones own decision to be executed, OSN users may be affected their peers concerns and adjust their decisions accordingly. To incorporate such peer effects in the strategy, we formulate a model to simulate an emotional mediation among multiple co-owners. Our mechanism, called CAPE, considers the intensity with which the co-owners are willing to pick up a choice (e.g. to release a photo to the public) and the extent to which they want their decisions to be affected by their peers actions. Moreover, CAPE automatically yields the final actions for the co-owners as the mediation reaches equilibrium. It frees the co-owners from the mediation process after the initial setting, and meanwhile, offers a way to achieve more agreements among themselves.
\end{abstract}

Index Terms-Social Networks, Collaborative Access Control, Game Theory, Peer effects.

\section{INTRODUCTION}

Many Online Social Networks (OSNs) now offer users free storage to upload their photos ${ }^{1}$ online. In addition, these OSNs also provide tools for users to edit photos, stitch photos together, and even make slideshows and galleries. Besides, OSNs also allow users to tag persons in the photo. Tagging a person not only facilitates users to organize the photos, but also encourages photo-sharing in OSNs. For example, the Picasa Web Albums ${ }^{2}$, which has recently been integrated with Goog $e^{3}{ }^{3}$, will give the person being tagged permission to view the photo and share with others.

\footnotetext{
${ }^{1}$ In this paper, for ease of presentation, we use photo as a shared content Our method works for other shared content such as video and documents when the co-owners can be identified successfully.

${ }^{2}$ http://picasaweb.google.com/

${ }^{3}$ https://plus.google.com
}

However, if it is not properly managed, photo tagging may violate a person's privacy and/or lead to his embarrassment. This is because the person being tagged can further share the photo with others. Consequently, the original uploader will lose control over who can access the photo as it may become available for the entire Web to view or be disseminated via Google+ stream. In fact, many inadvertent users may not even realize the size of the audience as they tag people and share their photos with others. Although a user can detag himself from a photo, he cannot stop other tagged users from sharing it in their social networks.

The widespread concerns to protect user privacy have prompted OSNs to develop access control mechanisms [1]. These are largely designed based on relationships and topology of the social networks [2], [3], [4]. Unfortunately, the decision for regulating the access to the shared photo still rests solely on the uploader of the photo. As such, these access control mechanisms are unable to deal with the privacy concerns of other persons that may appear in the photo.

Intuitively, we can view all persons appearing in a photo as co-owners of the photo. Each of these co-owners can thus voice his opinion about who can have access to the photo. By developing a method that considers the privacy of all coowners, a collective decision on the access restrictions may be determined. However, everyone has his desired preference of sharing at the appropriate exposure level that he is most comfortable with. It is thus not uncommon that conflicts will arise as a result of differing privacy preferences - while one may be excited about sharing his photo, another may prefer to keep it from public view. How to resolve such conflicts in differing privacy concerns and to support a fair collective decision-making strategy is an open problem.

We also notice that even though the users are very concerned about the privacy, they seldom do much to protect their privacy. In fact, users are just reluctant to spend time in specifying privacy policy. Thus, a practical OSN access control tool should be intuitive, light-weight, and automatic (i.e., require minimal human intervention/effort).

To this end, many researchers recently began to intro- 
duce collaborative access control policy-making mechanisms in OSNs [5], [6], [7]. These methods integrated the social relationship types and the topology of social networks in the policy-making, as well as in assessing the trust level of accessors. Simple voting functions (e.g. full-consensus, oneoverride, majority) are provided to deal with privacy conflicts. Moreover, the intensity of the user's perceived importance towards a specific preference also matters. For instance, Alice is essentially neutral and do not have any preference on whether to keep the photo private or share with the public; on the other hand, John may be very passionate (and hence has a higher level of intensity than Alice) about sharing photos to the public. Thus, intensity shall also be incorporated into the expression of user preference in access control rules. To promote fairness and truthfulness among users, a more sophisticated voting method was proposed in [5] to remove the incentive to conceal the true perceived intensity of a preference.

However, a thoughtful strategy should not only collect each individual's own intention, but also take into account the social interaction among the co-owners in the social network. Coowners of a photo are typically not business competitors where they need to hide their true intention and compete with one another to achieve maximum gain. Instead, they are likely to be friends/acquaintances/colleagues and hence there is a tendency to be considerate and sensitive to the feelings of one another. Consider the scenario where two close friends, Alice and Bob, had taken a photo together. Initially Bob wants to share this photo with other friends, whereas Alice is strongly against making the photo public. By taking Alice's feeling into consideration, Bob is likely to respect her and change his mind, hence achieving the consensus to keep this photo private. It is inevitable that peers exert tremendous influence on individual behaviors, let alone the ubiquitous interactions on OSNs. Such peer effects can be found in a vast literature in the field of sociology and psychology (e.g. [8], [9], [10], [11], [12]).

By taking into account peer effects in making collaborative access control rules, some conflicts of co-owners' intention will disappear naturally. We aim to treat everyone's preference with equal importance so that no single person's personal preference directly dominates the collective decision. At the same time, our proposed strategy, called CAPE, incorporates peer effects, allowing users to adjust their intention according to their neighbors' actions. The goal is to try to achieve more agreements, or even better, full consensus and satisfy everyone's privacy concerns. This is inherently different from collusion which has a negative connotation. In fact, considering peer effects on network may undermine colluding behavior. This is the case as a subgroup of users' decisions do not necessarily directly dominate the entire group's decision. The result of our strategy depends on the overall network structure of peer effects, that is, how each individual reacts to his neighbors.

In this work, we employ a game theoretic model to simulate the continuous decision adjustments that occur in social interactions. The theoretic model guarantees a unique equilibrium under appropriate parameter setting, which ensures the mediation will terminate. In the model, each player (which is a co-owner) expresses his own preference and his perceived peer effects independently. Each personal setting is private (i.e. no other players know his setting) and will be managed by the central strategy mechanism engine. Moreover, the model offers a direct solution, a "payoff-maximum" action, for each user, automatically. Thus, everyone will be satisfied with the action chosen by such a procedure. At the same time, except the initial set-up, we free the users from any effort and time during the mediation process.

The rest of this paper is organized as follows. In the next section, we introduce some preliminaries. In Section III, we discuss the challenges in designing the game theoretic collaborative access control model, and give a big picture of our solution. Section IV presents the setup phase for players (aka co-owners), and Section V shows the mediation procedure. In Section VI, we discuss several related issues. Section VII reviews some existing works, and finally, we conclude in Section VIII with directions for future work.

\section{REPRESENTATION OF OSNS}

In this section, we introduce the representation of an OSN. Roughly, we can categorize OSNs into two types: distancebased network and circle-based network. The former one classifies the users based on the topological distance. For example, hop 1 corresponds to Friends, hop 2 to Friends of Friends and hop $+\infty$ to Public. The latter one focuses on the specific classification of an individual's friends as groups, say Family, Colleague, School-mates. We first illustrate our work on distance-based network. We will discuss how our work can be extended to circle-based network in Section VI-C. Now, we shall introduce the core parts of a distance-based OSN and a few notations used in our proposed collaborative access control framework as follows:

1) $U$. The set of OSN users. Assume that each user $u_{i} \in U$ has a unique id, $i$.

2) $E$. The set of edges that connects the users. An edge $e \in$ $E$ connects two users, which can be either undirected or directed.

3) $d_{i j}$. Distance from $u_{i}$ to $u_{j}$, which can be measured with the path length.

4) Originator. The user who initiates the collaboration. In OSNs the originator is the user who first uploads the photo in his web album for sharing and tags other users who also appear in the photo.

5) Co-owner. The user who appears and has been tagged in the photo.

6) Player. The user, either the originator or the co-owner, who participates in the collaboration to make collective access control rules. We use the player $i$ and the player $u_{i}$ interchangeably in this work when no ambiguity arises.

7) Access Control Policy Choice Set, $\mathbb{C}$. On distanced-base OSNs, we can represent a control policy $c \in \mathbb{C}$ in terms 


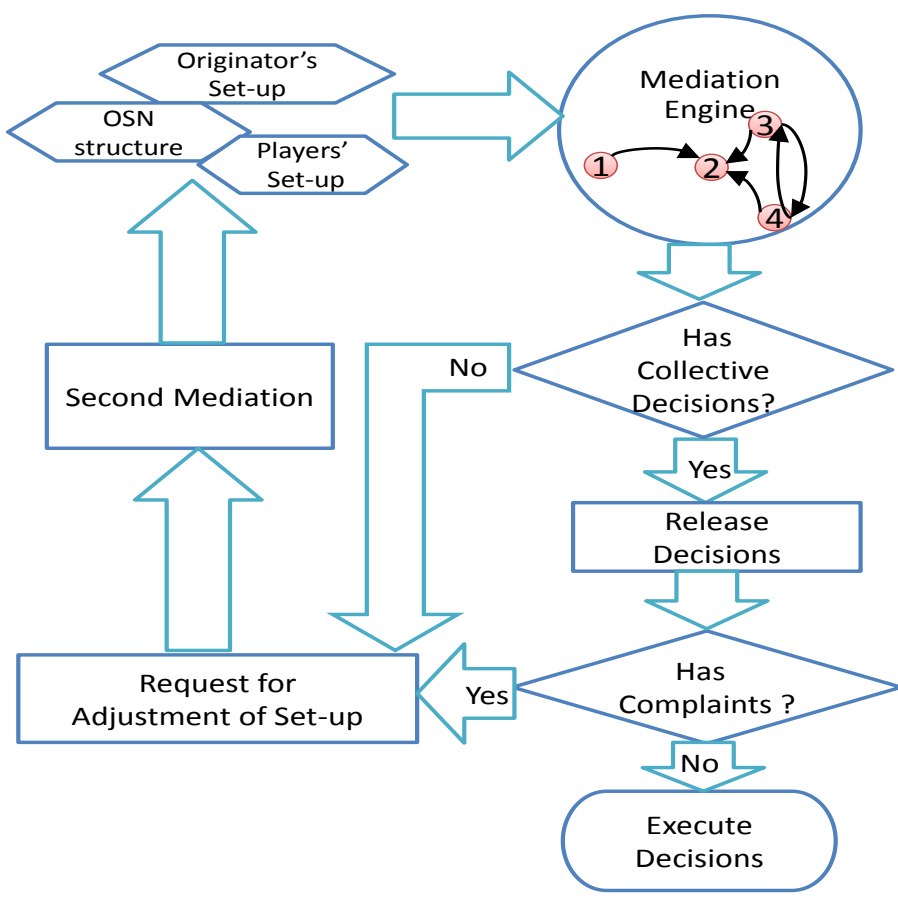

Fig. 1. The CAPE Framework

of $d$. For instance, 0 indicates keeping the photo private, whereas $+\infty$ means to share the photo with the public. As in existing work [5], in this paper, for distancebased OSNs, we consider a total of four options, namely, private (0), friends (1), friends-of-friends (2) and public $(+\infty)$.

\section{The Big Picture}

A straightforward method to make a collective decision in real life is to let each player explicitly show his preference first. After viewing other's actions, the players can further revise their preference settings [10], [11], [12], disclose their new decisions, and so forth until a common decision is reached after a few rounds. This is a typical scenario, as studied in a vast literature on sociology, where the behavior of an individual, say Alice, may change as she is being influenced by her peers.

Now, in our context, we can expect peer effects to come into play too. Among friends/colleagues, there will always be some who are more highly regarded and respected (or even feared); and opinions of such persons are likely to have a greater impact on others' decisions. For example, Alice may become more inclined to keep a photo private as a result of (some of) her neighbors' (aka friends and seniors) preferences to keep it private; on the other hand, the change in Alice's decision may have an impact on others for which she has influence over. Thus, we need a tractable formulation to incorporate such continuous interactions between the peers.

However, in real-life, OSN users often access the network independently and hence not all users will be online at the same time. Thus it is not practical and desirable for any access control mechanisms to require synchronization in time. In addition, the players may be stuck in an endless task since individuals can always adjust their decisions. To this end, we propose a method that simulates the negotiation and interaction among players, while, at the same time, ensures the simulation will terminate under an appropriate set-up. Specifically, we suggest a mediation procedure that facilitates the following features:

1) Each individual, say Alice, can perform an initial set-up independently (of course, the settings can be updated whenever it is necessary). Essentially, Alice assigns weights to her neighbors to reflect the degree of influence in which her neighbors have over her decision. There is no synchronization required in mediation process, as long as Alice sets up the initial configuration. In addition, Alice does not need to be personally involved in the mediation process, freeing her from the burden of mediation and saving her time.

2) The method should allow Alice to always choose the action that benefits her emotion most. In other words, after considering both her personal willingness and the peer effects, the method should always take the most appropriate strategy from Alice's perspective. We refer to this action as the maximum "emotional payoff" action.

3) The strategy should guarantee a unique Nash equilibrium. That is, briefly, the game should always reach a scenario where no player has the incentive to change only his own decision.

As we shall see, our proposed method ensures the above features, provided that each player should not regret the choice he has made in response to the actions taken by other players.

We are now ready to give an overview of our proposed framework - the CAPE framework that facilitates Collaborative Access control by considering Peer Effects. Our CAPE framework is depicted in Figure 1. The mediation engine, which is the key component, requires input from several sources: (a) OSN structure. The subgraph of the OSN that involves the co-owners/players; (b) Originator. The originator triggers the mediation process by uploading the photo and tagging the players; (c) Player. For each player, two types of information are provided. The first is contentdependent, i.e., his inclination (which we refer to as intensity towards the access control policies; this may be different for different shared content, e.g., Alice may be fine with making public a group photo, while she may not want to share the photo where she felt she may be embarrassed (e.g., she was drunk and was throwing out). The second is peer-effects-based, which specifies the player's inclination to be influenced by his immediate neighbors. We refer to this inclination as the peer effects. This information is more stable. It can be specified once during set-up, and only updated when necessary. In this paper, the mediation engine only considers how a player is influenced by his direct neighbors; moreover, the specification 
of the degree of influence must be positive. This is because our work models the situation of a constructive environment where players mutually support, or reinforce each other. We shall discuss this further in Section IV.

To initiate the mediation process, the originator uploads a photo, and invites the players (other users appearing in the photo) by tagging them. Based on the the social network structure and the players' specification, the mediation starts to simulate the continuous interaction between the players. The mediation process is done for each possible choice independently. In fact, for each choice, an unique equilibrium exists as long as the set-up's configuration meets the required conditions, and then the system can automatically compute the final intensity each player would like to select on the given choice. Once the final intensity of all the choices are ready, the choice with the highest intensity is considered to be the final choice the player would like to select. As each player's final decision is ready, the system will try to make a collective decision with a voting function. All the players will be informed of the outcome if any. The whole procedure terminates if the mediation succeeds and no complain arises. In the event where there is no equilibrium, and/or some players find the collection decision unacceptable, the players may have to adjust their inclinations for another round of mediation.

In this work, since the whole procedure aims to achieve more agreements, we suggest two voting functions: (i) full consensus, mediation succeeds only when all the players agree on the final decision; (ii) strong majority with a threshold $\theta$, that is, no fewer than $\theta$ percentage of players agree on the final decision. But for ease of discussion, we only consider full consensus as the voting function in all the examples. We shall discuss the mediation process and the voting function in Section V.

\section{Player Setup}

In our CAPE framework, we need each player to specify his preferences for the available choices, as well as the degree at which he may be influenced by his immediate peers' decision. We capture these with two types of variables:

- Intensity Score (I-Score) $x_{i}\left(c_{k}\right)$, which measures the inclination/extent to which the player $i$ is willing to take the choice $c_{k}$. Its value is unbounded and non-negative. However, to make it more intuitive for the users, in the initial set-up, we restrict $x_{i}^{0}\left(c_{k}\right)$ to be an integer and let $x_{i}^{0}\left(c_{k}\right)$ be between 0 and 5 . This essentially corresponds to six attitudes: \{strongly disagree, disagree, slightly disagree, slightly agree, agree, strongly agree $\}$. In this way we let all the players specify the values on the same ground. During the mediation peer effects cause $x_{i}\left(c_{k}\right)$ to change (increase) iteratively. So $x_{i}\left(c_{k}\right)$ is no longer bounded within the range $[0,5]$. But the player is only required to assign the initial values of his own I-Scores, the vector $\mathbf{x}_{i}^{0}$ for all the choices.

- Peer effects Score (PE-Score) $w_{i j}$, which characterizes how much weight the player $i$ intends to place on player $j$ 's action. In this work, we require $0 \leq w_{i j} \leq 1$. This ensures the model is a game of strategic complements where each player mutually supports one another.

\section{A. Setting I-Score}

To make an intuitive interface for users, we recommend presenting players the slide bars as tools for specifying the values. We propose two ways to let an user set his I-Scores, as pictured in Figure 2a and Figure 2b. The first one considers four independent choices: private, friends, friends of friends, public, where $d=0,1,2,+\infty$ respectively. This method allows players to set their I-Scores arbitrarily, i.e., players can set any values for the weights on different choices. A higher intensity score indicates the player has a stronger desire to take the choice. Referring to the setting in Figure 2a, the player essentially says: I don't quite agree to share the photo with the world; I sort of prefer to keep it private; however, I would most agree if we restrict the access to just friends or friendsof-friends. In the case where a player set all the I-Scores to be of the same value, we assume the player is willing to undertake any of such actions, regardless of their initial values (i.e., a player cannot reject all the possible actions together). As we shall see shortly, during the mediation process, each of these choices/options will be considered independently. For ease of reference, we shall refer to this method as Method $\mathbf{O O}$ (for 'option only').

Alternatively, we can design the bars as in Figure 2b, where it shows complementary assessment of "Take the choice or not". As depicted in Figure 2b, we consider all the choices except "private". Given a specific choice $c$, we consider two actions in turns, "Take $c$ " and "Against $c$ ". For example, the length of blue bar on the top indicates the intensity of picking the choice of "Friend(1)", whereas the red bar beside shows the intensity of being against the choice "Friend(1)". If a player is against all the three choices, it implicitly indicates he would like to keep the photo private. We shall discuss in Section V how these two complementary actions are independently considered during the mediation process. In other words, we actually have three pairs of choices to work with in the mediation process. We shall refer to this method as Method OC (for 'option and its complement').

Before leaving this section, we note that the flexibility of arbitrary setting has both pros and cons. On one hand, it allows a player, say Alice, to specify her preferences. For example, a player can specify a value of 5 for 'friends' and 0 for all the other options, indicating that he only want to restrict to friends, and nothing else. However, the flexibility may also lead to some undesirable settings, e.g., it does not seem to make sense to have a setting of 5 for 'friends-of-friends' and 0 for all other choices. Such flexibility requires users to fully appreciate the consequences of their settings (in order to ensure the settings are meaningful). Moreover, such flexibility also makes it more challenging for all users to reach a consensus.

To achieve more agreements easily, we can enforce some constraints on the setting. One reasonable approach is to assume that, if a player $i$ set $x_{i}^{0}\left(c_{k}\right)$ as the I-Score of a choice $c_{k}$, then the I-Scores of other more restricted preferences, except 


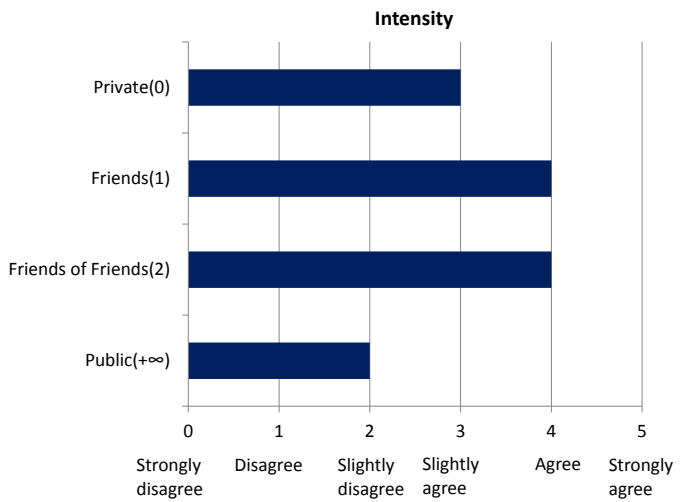

(a) Option Only(OO)

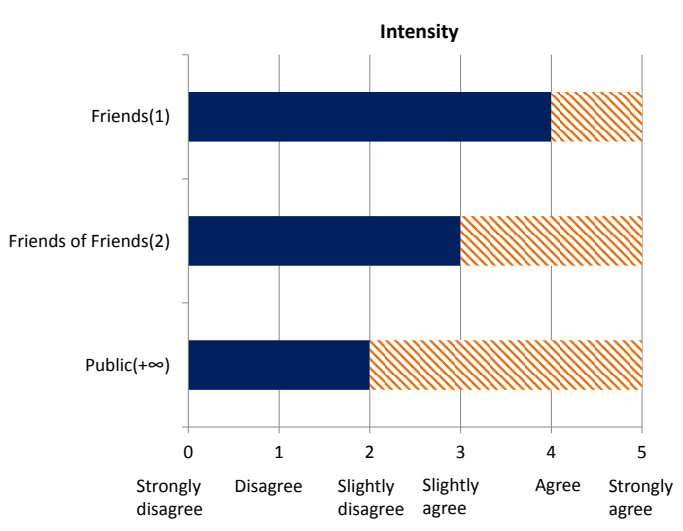

(b) Option and its Complement(OC)

Fig. 2. Two Designs of Intensity Bar

"private", must not be less than $x$. For instance, if a player slightly agrees with the choice "friends of friends", he must at least also slightly agree with the more restricted choices like "friends only". Such a constraint is reasonable presumed that, if one already agrees on a relatively relaxed choice, he cannot be against more private choices. This strategy is akin to asking players to conform to the group by sacrificing the joy of sharing and encouraging them to protect privacy.

\section{B. Setting PE-Score}

We note that the PE-Score needs to be set only. For each player, he essentially maintains an array of the PE-Score for each of his neighbors. All players could have set the PE-Scores for their friends when they first include them as friends. In the event that a player did not provide enough information, the default setting is assumed to be 0, i.e., he will not support others' options.

\section{The Mediation Process}

We now describe the mediation process which is an iterative procedure to simulate the social interaction among the players. To see our approach in action, let us first illustrate the whole procedure with a running example, and then present the proposed mediation mechanism.

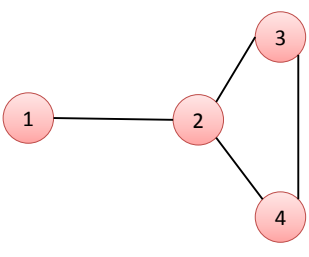

(a) Social Network

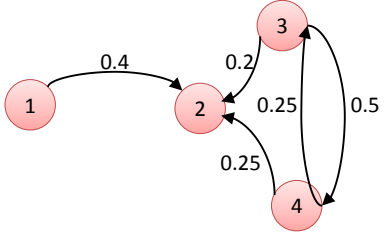

(b) Peer effects Network
Fig. 3. Peer effects in OSN

TABLE I

INITIAL I-SCORES WITH METHOD OO

\begin{tabular}{|c|c|c|c|c|c|}
\hline & Private & Friends & Friends of Friends & Public & Intention \\
\hline$u_{1}$ & 2 & 3 & 3 & $\underline{4}$ & Public \\
\hline$u_{2}$ & $\underline{5}$ & 0 & 0 & 0 & Private \\
\hline$u_{3}$ & 2 & $\underline{4}$ & 2 & 2 & Friends \\
\hline$u_{4}$ & $\underline{5}$ & 4 & 1 & 1 & Private \\
\hline
\end{tabular}

\section{A. An Example}

Consider a scenario where Player $u_{1}$ is a friend of Player $u_{2}$, and Player $u_{2}, u_{3}, u_{4}$ are colleagues. Figure 3 a shows the social network of their relationships in a graph model. Suppose the four of them have taken a photo together. The originator $u_{1}$ posted this photo on his own web album, and also wanted to share it on OSNs. So $u_{1}$ tagged all other users on this photo, trying to make a collective decision on whether this photo should be posted for public view or be kept private. Since we have only discussed the "full consensus" voting function, we shall illustrate the mediation process with this. Further, let us assume that each player has assigned the Iscores and the PE-Scores. For ease of presentation, we assume users specify their initial I-Scores using Method OO, i.e., each player specifies his preference for each option. We defer the discussion when the second method, Method OC, is used to Section V-C. Table I and II show the players' assigned values. The value underlined in Table I corresponds to the action the player prefers most in the beginning. As it turns out, player 2 is strongly concerned about his privacy over this photo than the others, and he is not going to change his mind according to others' intention. In contrast, player 1 is more willing to put the photo online; and meanwhile, he also values player 2's feeling and/or opinion.

From the setting, we observe that conflicts exist in the initial intention among the players. But through the mediation process, as we shall see later, we can derive each player's final intensity scores and their final action when the continuous interaction terminates, as shown in Table III. We note that $u_{1}$ turns out to have the same score (of 4) for both Private and Public. As such, we can pick either option. By selecting "Private", conflicts can be resolved, i.e., through the mediation, a full consensus on keeping this photo private has been reached. 
TABLE II

PEER EFFECTS SCORES

\begin{tabular}{|l|c|c|c|c|}
\hline & Player 1 & Player 2 & Player 3 & Player 4 \\
\hline Player 1 & 0 & 0.4 & 0 & 0 \\
\hline Player 2 & 0 & 0 & 0 & 0 \\
\hline Player 3 & 0 & 0.2 & 0 & 0.5 \\
\hline Player 4 & 0 & 0.25 & 0.25 & 0 \\
\hline
\end{tabular}

TABLE III

I-SCORES AT EQUILIBRIUM WITH METHOd OO

\begin{tabular}{|c|c|c|c|c|c|}
\hline & Private & Friends & Friends of Friends & Public & Intention \\
\hline$u_{1}$ & $\underline{4}$ & 3 & 3 & $\underline{4}$ & Public/Private \\
\hline$u_{2}$ & $\underline{5}$ & 0 & 0 & 0 & Private \\
\hline$u_{3}$ & $\underline{7}$ & 6.86 & 2.86 & 2.86 & Private \\
\hline$u_{4}$ & $\underline{8}$ & 5.71 & 1.71 & 1.71 & Private \\
\hline
\end{tabular}

\section{B. The Mediation Engine}

We shall now present the mediation engine used in our work to deal with conflicts that may arise in initial settings. As mentioned, our mediation factors in the peer effects of players. Our scheme is based on a game model, a variation of the game model of Ballester, Calvó-Armengol, and Zenou [13], which is also discussed in Chapter 9 of Jackson's book [9]. In this model, we use the variable payoff to describe to what extent the player considers a specific adjustment of his I-Score is appropriate in response to the actions of his neighbors. That is, the higher this "emotional" payoff is, the more the player assesses the appropriateness of this adjustment of I-Score. The variable payoff $p_{i}$ of the player $i$ is defined as follows:

$$
p_{i}\left(c_{k}\right)=a_{i} x_{i}\left(c_{k}\right)-\frac{b_{i}}{2}\left(x_{i}\left(c_{k}\right)\right)^{2}+\sum_{j \neq i} b_{i} w_{i j} x_{i}\left(c_{k}\right) x_{j}\left(c_{k}\right),
$$

where $a_{i} \geq 0$ and $b_{i} \geq 0$ are scalars, and $w_{i j}$ is the PE-Score value specified by player $i$. The expression $-\frac{b_{i}}{2}\left(x_{i}\left(c_{k}\right)\right)^{2}$ is a force to draw back to player $i$ 's own decision. It is easy to see that a high intensity of the player $i$ 's own intention will tend to inhibit the increase of the payoff. Therefore, player $i$ can see some trade-off by taking further action to adjust his I-Score. Moreover, since $w_{i j} \geq 0$, the payoff tends to increase by considering other's I-Score, $x_{j}\left(c_{k}\right)$, which thus simulates the interaction where the players reinforce each other's actions. That is, when the intensity of the neighbors' action is high, the intensity of the player's corresponding action would also be high. Intuitively, it describes the phenomenon where an individual tends to conform to the patterns of his peers' behaviors.

We assume that each player always chooses the action that offers the highest "emotional payoff", and he can never regret the action he takes at each step. Then, we can derive such action to adjust I-Score by setting the derivative of the payoff $p_{i}\left(c_{k}\right)$ to 0 . Hence, such payoff-maximizing action can be described by

$$
x_{i}\left(c_{k}\right)=\frac{a_{i}}{b_{i}}+\sum_{j \neq i} w_{i j} x_{j}\left(c_{k}\right)
$$

Equation (2) indicates that player $i$ should continuously adjust

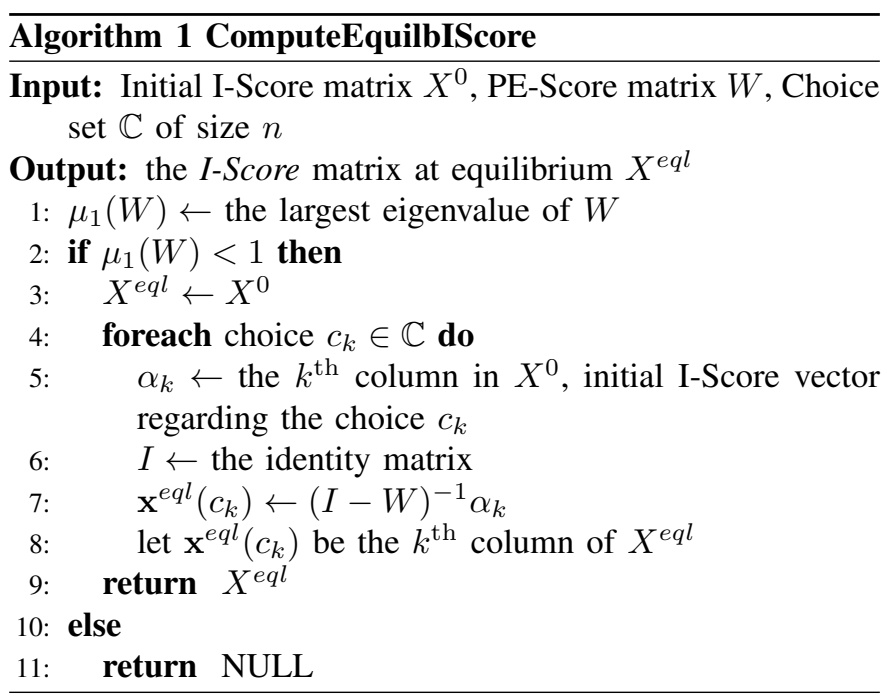

his I-Score regarding other player's updated I-Score $x_{j}\left(c_{k}\right)$ in each round. But, in fact, the further user intervention is not needed, because the final I-Scores at equilibrium state can be directly derived with an analytical method. Therefore, we can directly compute the final action for each player based on just the initial setting. To illustrate such analytical solution, let us first denote $\mathbf{x}^{e q l}\left(c_{k}\right)$ as the vector solution of such IScore $x_{i}\left(c_{k}\right)$ at the equilibrium. And let $\alpha_{k}$ be the vector of $\frac{a_{i}}{b_{i}}$ regarding the choice $c_{k}$. Then the vector solution can be expressed as follows,

$$
\mathbf{x}^{e q l}\left(c_{k}\right)=(I-W)^{-1} \alpha_{k},
$$

where $I$ is the identity matrix. Since $b_{i}$ is a scalar, we can set $b=b_{i}=1$ for all $i$. Correspondingly, $\alpha_{k}$ is set to be $a_{i} . W$ is the matrix whose entry is the PE-Score, $w_{i j}$. We can further think of $W$ as a weighted and directed network, the peer effects network as depicted in Figure $3 b$, where the weight of edge is assigned to be $w_{i j}$.

In Equation (3), the matrix $(I-W)^{-1}$ serves as the factor of peer effects, applied on the vector $\alpha_{k}$. At the very beginning, without factoring peer effects, we can consider $\alpha_{k}$ to be just the vector containing all the initial I-Score regarding the choice $c_{k}$. Thus, we can set the entry of $\alpha_{k}, a_{i}$, to be $x_{i}^{0}\left(c_{k}\right)$.

With Equation (3), we can compute the final I-Scores directly. Algorithm 1 describes the entire procedure. The above solution holds if $I-W$ is invertible and $(I-W)^{-1}$ is nonnegative. Ballester et al. [13] shows that these conditions can be met if and only if $\mu_{1}(W)<1$, where $\mu_{1}(W)$ is the largest eigenvalue of $W$. Another sufficient condition to satisfy the conditions is to let all $w_{i j} \geq 0$, and the sum of the entries of each row/column of $W$ be less than 1 .

With the aboved formula, we can automatically compute the intensity scores of all the choices for each player as the mediation reaches equilibrium. The choice with the highest score will be selected as the final action that the player would like to undertake. Formally, the final decision $\gamma_{i}$ of player $i$ 
TABLE IV

INITIAL I-SCORES WITH METHOD OC

\begin{tabular}{|c|c|c|c|c|}
\hline & Friends & Friends of Friends & Public & Intention \\
\hline$u_{1}$ & 5 & 5 & 4 & Public \\
\hline$u_{2}$ & 3 & 3 & 0 & Friends of Friends \\
\hline$u_{3}$ & 4 & 4 & 0 & Friends of Friends \\
\hline$u_{4}$ & 2 & 2 & 2 & Private \\
\hline
\end{tabular}

TABLE V

I-SCORES AT EQUILIBRIUM WITH METHOD OC

\begin{tabular}{|c|c|c|}
\hline & Public & Against Public \\
\hline$u_{1}$ & $\underline{4.0}$ & 3.0 \\
\hline$u_{2}$ & 0 & $\underline{5.0}$ \\
\hline$u_{3}$ & 1.14 & $\underline{9.29}$ \\
\hline$u_{4}$ & 2.29 & $\underline{6.57}$ \\
\hline
\end{tabular}

(a)

\begin{tabular}{|c|c|c|}
\hline & Friends of Friends & Against Friends of Friends \\
\hline$u_{1}$ & $\underline{6.2}$ & 0.8 \\
\hline$u_{2}$ & $\underline{3}$ & 2 \\
\hline$u_{3}$ & $\underline{6.83}$ & 3.6 \\
\hline$u_{4}$ & $\underline{4.46}$ & 4.4 \\
\hline
\end{tabular}

(b)

is,

$$
\gamma_{i}=\arg \max _{c_{k} \in \mathbb{C}} x_{i}\left(c_{k}\right)
$$

In Method OO, we compare a player's I-Scores of all the choices together and select the one with the highest score to be his final decision. Algorithm 2 illustrates this procedure.

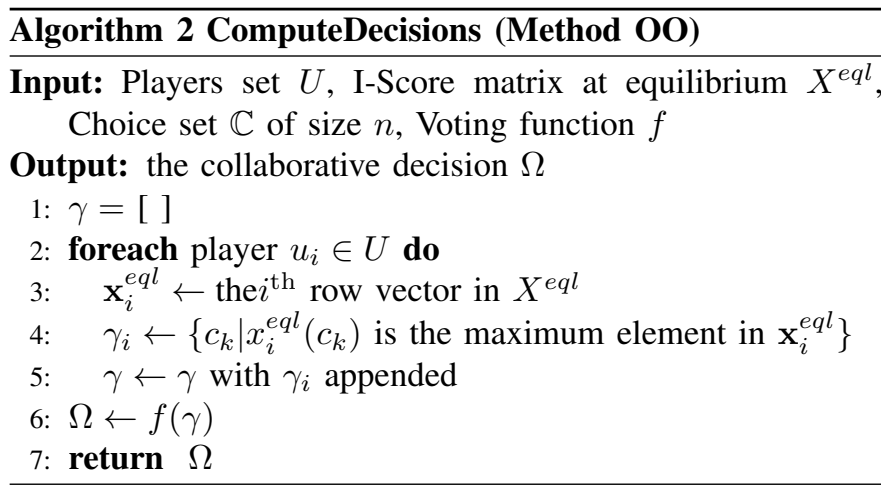

\section{Constraining the I-Score Setting}

Now, it is possible that with more choices/options and arbitrary setting of I-Score values, the chances of achieving agreements decreases. A solution to this problem, as described in Section IV, is to be "biased" towards privacy by restricting users to always specify equal or greater intensity scores for more restricted choices. To see this explicitly, we use the second method, Method OC, to illustrate. Table IV shows an example of user settings satisfying the above constraints. We shall first consider whether all the players agree on the preference "Public". Recall that under Method OC, each choice results in two actions, and they are to be mediated independently; and the final decision for the choice is determined by the action with the larger I-Score. Table V(a) shows

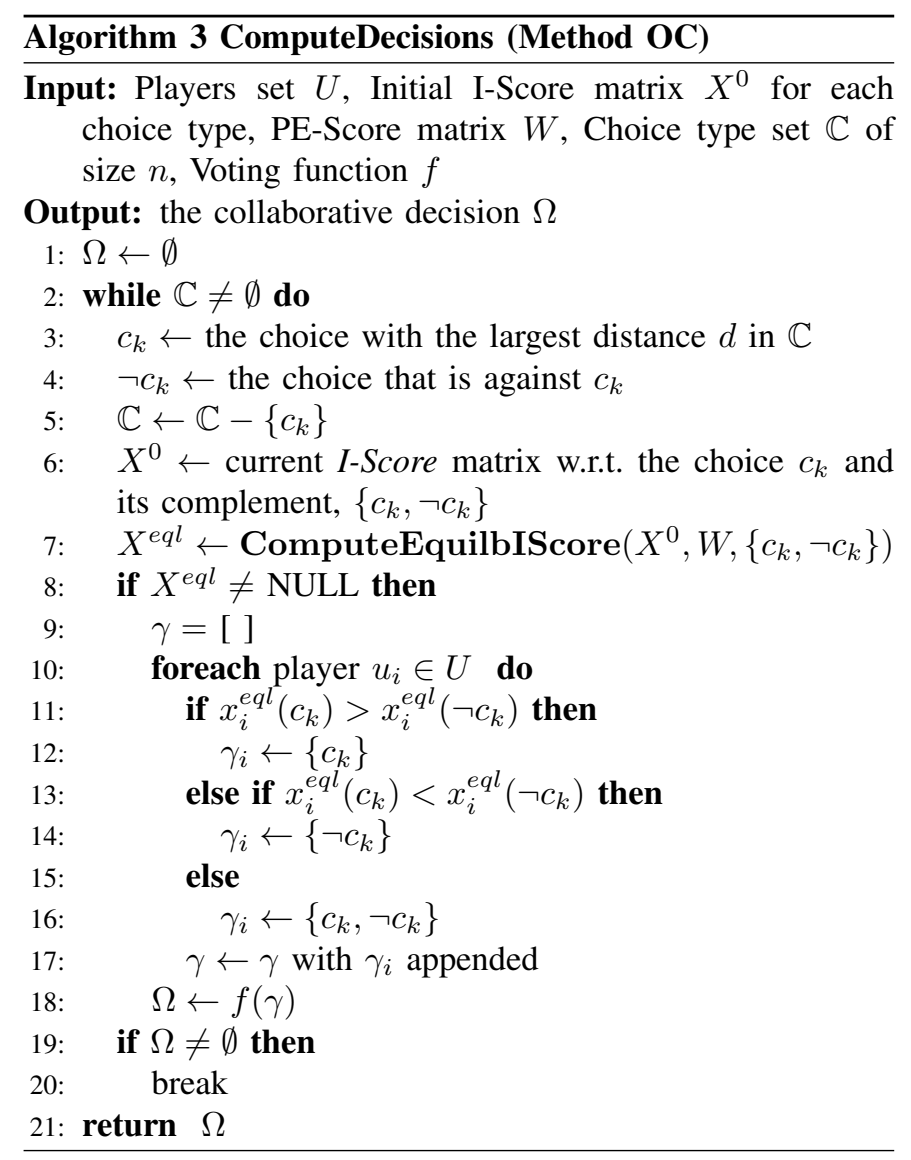

the result of the mediation process for "Public" and "Against Public". As shown in Table V(a), $u_{1}$ prefers "Public" while the rest vote for "Against Public". Since there is no full consensus, we continue to consider the next pair of choices, "Friends of Friends" and "Against Friends of Friends". This time, as shown in Table $\mathrm{V}(\mathrm{b})$, all the players agree on the choice "Friends of Friends". So the mediation stops here and make the final decision as sharing this photo within the distance no more than "Friends of Friends". The algorithmic description of the procedure is given in Algorithm 3.

\section{DISCUSSION}

In order for our CAPE framework to be developed into a full-fledge robust solution for practical use, there are several issues that need to be addressed. Here, we shall focus on three of them: (a) How to guide the players to configure the set-up; (b) How to facilitate second mediation in order to achieve more agreements; and (c) how to extend our work to circlebased OSNs. We will discuss each of these in the following subsections.

\section{A. Configuring the set-up}

One of the key parameters in the CAPE framework is the setting of the players' PE-Scores, i.e., how each player views the influence of his peers over his decision. However, the framework is meaningful only if an equilibrium exists. In 
particular, equilibrium exists and is unique when the PE-Score matrix is not overly dense. The PE-Score matrix is dense when the players over-rely on each other's decision. For example, every player may want the opinion of every other player. As a result of such cross-effect, the intensity of a player's choice is always positively reinforced by other players, which in turn leads to an unbounded increase in the intensity of individual's action (and thus the model cannot reach an equilibrium state).

Therefore, it is important to guide the players to assign or adjust their PE-Score values to ensure an equilibrium state. Overall, a guideline for the players is to assign the PE-Score moderately. In fact, in our framework, we have restricted the PE-Score to direct neighbors, i.e., a player only provide the PE-Score for his immediate neighbors. This helps to reduce the chance for large cross effect (since the PE-Score matrix becomes more sparse). However, even with this restriction, it is still possible that equilibrium cannot be reached. In fact, when the PE-score matrix contains a column, say $\mathrm{Col} j$, and its corresponding row, say Row $j$ such that the sums of the values of Col $j$, and Row $j$ are both greater or equal to 1 , then no equilibrium can be reached. Intuitively, this can happens when player $j$ is very prominent among the other players, and, at the same time, player $j$ also tends to respect his followers' opinions. A solution to handle this case is to let player $j$ dominates his own decision (i.e., ignore other players peer effects), preventing the existence of feedback loops. To see this in action, let us consider the following extreme case.

Example 1. Consider the scenario where a professor and his four students took a photo together. All of the students respect the professor's opinion. However, at the same time, the professor also decides to conform to his students' choices, as shown in Table VI. The mediation cannot proceed due to the very large cross effect between the professor and his students. It can be observed that both Row 1 and Col 1 are greater or equal to $1 . \mu_{1}(W)$, the current greatest eigenvalue of the PE-Score matrix, is 1 , which does not satisfy the condition for existence of the equilibrium. One possible solution to this case is to let the professor reduce his dependency on his students, like making a adjustment as showed in Table VII. Table VIII and Table IX show the mediation outcome after such adjustment.

TABLE VI

PE-SCORES BEFORE ADJUSTMENT

\begin{tabular}{|c|c|c|c|c|c|}
\hline & Prof & $s_{1}$ & $s_{2}$ & $s_{3}$ & $s_{4}$ \\
\hline Prof & 0 & 0.25 & 0.25 & 0.25 & 0.25 \\
\hline$s_{1}$ & 1 & 0 & 0 & 0 & 0 \\
\hline$s_{2}$ & 1 & 0 & 0 & 0 & 0 \\
\hline$s_{3}$ & 1 & 0 & 0 & 0 & 0 \\
\hline$s_{4}$ & 1 & 0 & 0 & 0 & 0 \\
\hline
\end{tabular}

We thus develop the following heuristics to address this problem. Given the PE-Score matrix, we determine if the greatest eigenvalue of the PE-Score matrix is less than 1. If so, we expect the existence of an equilibrium. Otherwise, we try to find out a player $i$ such that the sum of the values
TABLE VII

PE-SCORES AFTER ADJUSTMENT

\begin{tabular}{|c|c|c|c|c|c|}
\hline & Prof & $s_{1}$ & $s_{2}$ & $s_{3}$ & $s_{4}$ \\
\hline Prof & 0 & 0.2 & 0.2 & 0.2 & 0.2 \\
\hline$s_{1}$ & 1 & 0 & 0 & 0 & 0 \\
\hline$s_{2}$ & 1 & 0 & 0 & 0 & 0 \\
\hline$s_{3}$ & 1 & 0 & 0 & 0 & 0 \\
\hline$s_{4}$ & 1 & 0 & 0 & 0 & 0 \\
\hline
\end{tabular}

TABLE VIII

INITIAL I-SCORES IN THE EXTREME CASE

\begin{tabular}{|c|c|c|c|c|c|}
\hline & 0 & 1 & 2 & $+\infty$ & Decision \\
\hline Prof & 0 & $\underline{4}$ & 0 & 0 & Friends \\
\hline$s_{1}$ & 3 & 3 & 3 & 3 & Any \\
\hline$s_{2}$ & 3 & 3 & 3 & 3 & Any \\
\hline$s_{3}$ & 3 & 3 & 3 & 3 & Any \\
\hline$s_{4}$ & 3 & 3 & 3 & 3 & Any \\
\hline
\end{tabular}

of row $i$ and column $i$ are greater than or equal to 1 , and request player $i$ to revise his PE-score. In particular, it has been recommended, as part of the sufficient condition for the existence of equilibrium, that the sum of each player's PEScore (the sum of each row) should be less than 1, unless he really wants to fully rely on the others' opinions. This process is repeated until an equilibrium can be reached.

\section{B. Second Round of Mediation}

Recall that our goal is to resolve the conflicts that arise in making collaborative decisions. So far, we have assumed that we can always reach a consensus that is acceptable to all players in one round of the mediation procedure. However, the mediation may fail. This happens when full consensus cannot be reached with regard to the set-up. It may also occur when not all players are satisfied with the collaborative decision derived from CAPE with a majority-mode voting function. In this section, we discuss how to further facilitate mutual collaboration if complaints about the outcome arise. The idea here is to identify a key player, say John, who has the highest effect on the aggregate outcome, and let the players who are not satisfied with the outcome turn to John for help. But notice that an unique feature of our method is that the final outcome closely depends on the peer effects network. Collusion is hard to succeed in such circumstance, since an individual or a small group, or even the key player John, does not necessarily dominate the result. Nevertheless, we can still encourage the players to approach John, who gets a lot of respect from his neighbors and has a high overall impact on the group, for help. Because such key player may easily persuade his followers to change their settings as well. In this way, the players can publicly request a second-chance mediation, instead of trying to employ colluding behaviors in private. Since John often gets more respect from his neighbors, it is more likely that the aggregate collective result be reduced optimally if he is willing to change his intention score. But bear in mind that this does not necessarily lead to a bending aggregate outcome. The outcome is still closely affected by the peer effects network. In addition, we suggest that all the players should have the 
TABLE IX

I-SCORES AT EQUILIBRIUM IN THE EXTREME CASE

\begin{tabular}{|c|c|c|c|c|c|}
\hline & 0 & 1 & 2 & $+\infty$ & Decision \\
\hline Prof & 12 & $\underline{32}$ & 12 & 12 & Friends \\
\hline$s_{1}$ & 15 & $\underline{35}$ & 15 & 15 & Friends \\
\hline$s_{2}$ & 15 & $\underline{\underline{35}}$ & 15 & 15 & Friends \\
\hline$s_{3}$ & 15 & $\underline{\underline{35}}$ & 15 & 15 & Friends \\
\hline$s_{4}$ & 15 & $\underline{35}$ & 15 & 15 & Friends \\
\hline
\end{tabular}

TABLE X

INTERCENTRALITY SCORES

\begin{tabular}{|c|c|}
\hline & Intercentrality \\
\hline$u_{1}$ & 1.96 \\
\hline$u_{2}$ & 1 \\
\hline$u_{3}$ & 3.8 \\
\hline$u_{4}$ & 2.7 \\
\hline
\end{tabular}

right to know who the key player is, whether there is anyone who has turned to the key player for help, and whether the key player agrees to adjust his intention or not. It should be a public procedure for petition for another round of mediation, which is distinguished from the colluding behavior (that are done in private).

In Ballester et al.'s work [13], they showed that the key player can be identified by ranking the players' intercentrality. Let $M=[I-W]^{-1}$, and $m_{i j}$ be its entry. $m_{i j}$ can also be written as $\sum_{k=0}^{+\infty} w_{i j}{ }^{k}$. This expression counts the number of weighted paths that start from $i$ and end at $j$. With the matrix $M$, we define the intercentrality of player $u_{i}$ as follows:

$$
\eta_{i}=\frac{\left(\sum_{j=1}^{n} m_{i j}\right)^{2}}{m_{i i}}
$$

The intercentrality actually "counts the total number of direct and indirect weighted paths that hit i" [13]. Briefly, it considers not only a player's centrality, but also his contribution to other's centrality.

Example 2. Consider a scenario where, using the second method, the player $u_{4}$ is not satisfied with the outcome "Friends of Friends" for he is really concerned about privacy. So $u_{4}$ asks the originator $u_{1}$ for a second mediation, and request to see who the key player is in their current peer effects network. Table $\mathrm{X}$ shows the intercentrality of each player. As it turns out, $u_{3}$ is the key player. Assume that $u_{4}$ talks to $u_{3}$, and persuades $u_{3}$ to change his intensity score. $u_{3}$ resets his set-up as ["Friends", 4], ["Friends of Friends, 2"], ["Public", $0]$. All the players are also informed that $u_{4}$ is not satisfied with the previous outcome and has asked the key player $u_{3}$ to reconsider his setting. If the originator agrees to set up a second mediation, all the players can reset their set-up and then a new mediation begins. Suppose, in this example, all the other players do not change their setting. The new result becomes the choice "Friends", as shown in Table XII [b], which further protects the player's privacy as a result of $u_{4}$ 's complaint.
TABLE XI

AdJusted Initial I-SCORES With Method OC

\begin{tabular}{|c|c|c|c|c|}
\hline & Friends & Friends of Friends & Public & Intention \\
\hline$u_{1}$ & 5 & 5 & 4 & Public \\
\hline$u_{2}$ & 3 & 3 & 0 & Friends of Friends \\
\hline$u_{3}$ & 4 & 2 & 0 & Friends of Friends \\
\hline$u_{4}$ & 2 & 2 & 2 & Private \\
\hline
\end{tabular}

TABLE XII

I-Scores at Equilibrium with Method OC IN the SeCond MEDIATION

\begin{tabular}{|c|c|c|}
\hline & Friends of Friends & Against Friends of Friends \\
\hline$u_{1}$ & $\underline{6.2}$ & 0.8 \\
\hline$u_{2}$ & $\underline{3}$ & 2 \\
\hline$u_{3}$ & 4.54 & $\underline{5.88}$ \\
\hline$u_{4}$ & 3.89 & $\underline{4.97}$ \\
\hline
\end{tabular}

(a)

\begin{tabular}{|c|c|c|}
\hline & Friends & Against Friends \\
\hline$u_{1}$ & $\underline{6.2}$ & 0.8 \\
\hline$u_{2}$ & $\underline{3}$ & 2 \\
\hline$u_{3}$ & $\underline{6.82}$ & 3.6 \\
\hline$u_{4}$ & $\underline{4.46}$ & 4.4 \\
\hline
\end{tabular}

(b)

\section{Circle-based Social Network}

In this part, we discuss how to extend our strategy to circlebased social networks. In circle-based social networks, users categorize their friends into different groups. We adapt the method developed by Hu et al [7]. Essentially, it is not practical to list out all the policy choices by taking into account all the player's circles together. Instead, we let each player considers the trust level of every accessor from his own perspective. Specifically, given an accessor, each player specifies the intensity in terms of his own circles to decide whether to grant access to this accessor. For example, given a photo, Alice may have the following settings for her circles: (Family, agree), (Labmates, slightly agree), (Strangers, disagree). Based on this setting, Alice's preference for the accessor depends on which circle the accessor belongs to. In our example, if Alice wants to share the photo with John and John is Alice's labmate, then Alice is essentially saying she is fine with sharing the photo with John. On the other hand, if John is a stranger to Alice, then Alice basically opted to keep the photo private. In some sense, what we really have is an implicit choice which is determined by setting of the circle which the accessor falls into. We can then derive the collaborative result based on each player's intensity score towards this given accessor.

Instead of taking the average aggregate decision as in [7], our strategy facilitates the players to adjust their decision towards the strangers outside their own circles by considering peer effects. This is based on the assumption that one would like to put more trust on a stranger as this stranger is also a friend of his friends. To see why this is useful, let us consider the following example.

Example 3. Consider the scenario where the player $u_{i}$ does not know the accessor $a$ personally. However, the accessor $a$ is in fact in the circle of one of $u_{i}$ 's friend, $u_{j}$. As $u_{i}$ is not 
familiar with $a, u_{i}$ cannot accurately assess the risk to share the photo with $a$. Alternatively, with our strategy, $u_{i}$ can refer to $u_{j}$ 's opinion since $u_{j}$ may know $a$ well. Note that $u_{i}$ even does not need to predict whether $a$ knows his friend $u_{j}$ or which exact extended circle $a$ belongs to. Since in circle-based networks, one is not likely to know the constituents of other's circles, our method helps the user get a better assessment of a stranger's risk by looking at others' actions.

\section{RELATED WORK}

Traditionally, OSNs have largely empowered the publisher of the content, say Alice, to be solely responsible for regulating access to shared content. As such, the research centers on determining the set of users who can have access to the data. Some models [2], [3] are based on the topology of the social networks, for example, in Facebook [3], Alice can restrict access to friends, friends of friends, groups or public; others rely on the relationships between the publisher and the accessors [14]; in addition, there are models [4], [15] that consider the trustworthiness and/or reputation of the users.

More recently, researchers have started to look at designing collaborative frameworks to support multi-party data sharing for OSNs [16], [5], [6], [7]. Squicciarini et al. [5] employed the Clarke Tax algorithm [17] as a voting strategy in their proposed model. The Clarke Tax strategy disincentivizes players to lie about the true valuation of their preference, and thus promotes truthfulness among users. However, the Clarke Tax voting strategy is vulnerable to bidder collusion [18]. The small number of players can collude and over expressing their preference to some extent. In this way they can bend the entire collective decision without paying the clarke tax. Besides, the final decision can be determined directly by only one "pivotal" individual, the one who is willing to pay more numerarie (e.g. tax, credit), whereas others with little numerarie just can not afford to influence the collective decision.

Carminati et al. [6] introduced an enhanced topology-based access control architecture by user collaboration. They also exploited semantic web technologies to support flexible representations of collaborator's relationships and resources. Hu et al. [7] formalized a multiparty access control model to address the same issue. Their proposed conflict resolution mechanism aggregates each player's decision policy and sensitivity towards a specific accessor and thus leverages each player's preference in collective decision-making. They also introduced methods to perform analysis (e.g. correctness analysis, authorization analysis) on the access control model. However, user's concern about privacy is not immutable. OSN users are connected and vastly influenced by their neighbors. Thus, we propose our method to simulate such social interaction automatically.

\section{CONCLUSION}

In this paper, we have revisited the problem of protecting user privacy in online social networks (OSNs). In particular, we have investigated the design of access control mechanisms for protecting shared content where co-owners may have differing and conflicting privacy preferences. A novel collaborative access control mechanism has been designed. Our key insight is that peer effects should be a key contributing factor to be considered in resolving conflicting preferences. Our proposed framework, CAPE, is based on graph theoretic model, and is able to lead to consensus that is acceptable to the co-owners. Our CAPE framework can be applied to both distance-based and circle-based networks. We also looked how the peer effects scores should be set to ensure equilibrium. Moreover, we also discussed how to handle the scenario when a player may not be satisfied with the outcome.

\section{ACKNOWLEDGMENT}

Qian Xiao is supported by a scholarship from the NUS Graduate School for Integrative Sciences and Engineering. The project is supported by a research grant R-252-000-433-305 funded by the Agency for Science, Technology and Research $(A * S T A R)$ in Singapore.

\section{REFERENCES}

[1] B. Carminati and E. Ferrari, Privacy-aware access control in social networks: Issues and solutions. Springer, 2010, pp. 181-195.

[2] P. W. L. Fong, M. M. Anwar, and Z. Zhao, "A privacy preservation model for facebook-style social network systems," in ESORICS, 2009, pp. 303-320.

[3] Facebook Privacy Policy, http://www.facebook.com/policy.php/.

[4] B. Carminati, E. Ferrari, and A. Perego, "Rule-based access control for social networks," ser. OTM'06, 2006, pp. 1734-1744.

[5] A. C. Squicciarini, S. Mohamed, and W. Joshua, "Privacy policies for shared content in social network sites," The VLDB Journal, vol. 19, no. 6, pp. 777-796, Dec. 2010.

[6] B. Carminati and E. Ferrari, "Collaborative access control in on-line social networks," in CollaborateCom, 2011, pp. 231-240.

[7] H. Hu, G.-J. Ahn, and J. Jorgensen, "Multiparty access control for online social networks: Model and mechanisms," IEEE Transactions on Knowledge and Data Engineering, vol. 99, no. PrePrints, 2012.

[8] S. Goyal, Connections: An Introduction to the Economics of Networks. Princeton, NJ, USA: Princeton University Press, 2007.

[9] M. O. Jackson, Social and Economic Networks. Princeton, NJ, USA: Princeton University Press, 2008.

[10] M. O. Jackson and X. Vives, "Social networks and peer effects: An introduction," Journal of the European Economic Association, 2010.

[11] J. W. Thibaut and H. H. Kelley, The social psychology of groups. Wiley, New York, 1959.

[12] Y. Bramoullé, H. Djebbari, and B. Fortin, "Identification of peer effects through social networks," Journal of Econometrics, vol. 150, pp. 41-55, 2009.

[13] C. Ballester, A. Calvó-Armengol, and Y. Zenou, "Who's who in networks. wanted: The key player," Econometrica, vol. 74, no. 5, pp. $1403-$ 1417, Sep. 2006.

[14] P. W. L. Fong, "Relationship-based access control: protection model and policy language," in CODASPY, 2011, pp. 191-202.

[15] S. R. Kruk, S. Grzonkowski, A. Gzella, T. Woroniecki, and H.-C. Choi, "D-foaf: Distributed identity management with access rights delegation," in $A S W C, 2006$, pp. 140-154.

[16] H. Hu, G.-J. Ahn, and J. Jorgensen, "Detecting and resolving privacy conflicts for collaborative data sharing in online social networks," in ACSAC, 2011, pp. 103-112.

[17] E. H. Clarke, "Multipart pricing of public goods," Public Choice, vol. 11, pp. 17-33, 1971.

[18] G. Weiss, Ed., Multiagent systems: a modern approach to distributed artificial intelligence. MIT Press, 1999. 